\title{
Vergleich der unspezifischen bronchialen Provokation mit Methacholin unter kontrollierter und freier Inhalation
}

\author{
Comparison of Unspecific Bronchial Provocation During Controlled \\ and Spontaneous Inhalation
}

Autoren

Institut
C. Wenzel ${ }^{1}$, P. Brand ${ }^{2}$, C. Herpich², S. Häußermann², T. Meyer², B. Müllinger ${ }^{3}$, G. Scheuch ${ }^{3}$, K. Häußinger ${ }^{1}$

Asklepios Fachkliniken München-Gauting, Gauting

Inamed Research GmbH\&Co. KG, Gauting

Activaero $\mathrm{GmbH}$, Gemünden eingereicht 12.12. 2005

akzeptiert 13.2. 2006

\section{Bibliografie}

Dol $10.1055 / \mathrm{s}-2006-932149$

Pneumologie 2006; 60;

467-471

(c) Georg Thieme Verlag KG Stuttgart · New York.

ISSN 0934-8387

Korrespondenzadresse

Dr. Peter Brand

Inamed Research

$\mathrm{GmbH} \&$ Co. KG

Robert Koch-Allee 29

82131 Gauting

Brand@inamed.de

\section{Zusammenfassung}

Die Verwendung von kontrollierten Atemmanövern bei der Inhalation von Medikamenten zeichnet sich durch eine hohe Dosis-Reproduzierbarkeit aus, die auch bei der Durchführung von bronchialen Provokationstests von Vorteil sein kann. In der vorliegenden Studie wurde bei 30 Probanden mit Atopie-Anamnese in einem crossover Design je ein bronchialer Provokationstest mit Methacholin und dem Viasys-Jäger-APS sowie mit kontrollierter Inhalation (AKITA-System) (vorgegebenes Inhalationsvolumen und -fluss) durchgeführt. Gemessen wurde die Häufigkeit positiver Befunde im Studienkollektiv, wobei „positiv“ entweder als 20\%-iger Abfall des $\mathrm{FEV}_{1 \text {, }}$ oder als $100 \%$-Anstieg des sRaw definiert war. Es zeigten sich keine signifikanten Unterschiede in der Prävalenz positiver Testergebnisse zwischen beiden Methoden: APS-FEV 1 :8, AKITA-FEV 1 :9; APS-sRaw: 18, AKITA-sRaw: 17. Es reagierten also erheblich mehr Personen mit einem 100\%-igen Anstieg des sRaw, als mit einem Abfall des $\mathrm{FEV}_{1}$. Allerdings hat sich gezeigt, dass zwischen den Ergebnissen der beiden Methoden Diskrepanzen bestehen. Nur in 25 (sRaw: 21) von 30 Fällen stimmte das Ergebnis (positiv oder negativ) zwischen beiden Methoden tatsächlich überein. Trotz dieser Diskrepanzen kann gefolgert werden, dass das AKITA-System mit kontrollierter Inhalation von Methacholin eine Alternative zum APS-System darstellt.

\section{Abstract}

Using controlled breathing patterns during inhalation of drugs is characterized by a high dose reproducibility which may be of advantage for bronchial provocation testing. In this study 30 healthy subjects with an anamnesis of atopy underwent in a randomized cross-over design bronchial provocation testing with methacholine either with the Viasys-Jäger-APS system or with controlled inhalations (AKITA-System) (controlled inhalation volume and flow). Measured was the frequency of positive test results. Positive test results were defined by a $20 \%$ decline of $\mathrm{FEV}_{1}$ or a $100 \%$ increase of specific airway resistance (sRaw). There were no significant differences in the prevalence of positive test results obtained with both techniques: APS-FEV $1: 8$, AKITA-FEV 1 : 9; APS-sRaw: 18, AKITA-sRaw: 17. More subjects showed a $100 \%$ increase of sRaw as compared to a $20 \%$ decrease of $\mathrm{FEV}_{1}$, which may be interesting in order to understand differences in the diagnostic information given by both parameters. However, there were some discrepancies: only in 25 of 30 cases (sRaw: 21 of 30 cases) the results (positive or negative) agreed between both techniques. Although the two techniques for bronchial provocation test showed some discrepancies, these data suggest that controlled inhalations may be an alternative to the APS-system.

\section{Einleitung}

Die bronchiale Hyperreagibilität gegenüber unspezifischer Provokation, kalter Luft oder Anstrengung findet man bei einer Reihe von Personengruppen [1-4]: Patienten mit bronchialem Asthma, COPD, bei Atopikern, aber auch bei gesunden Personen, die Umweltnoxen ausgesetzt sind, oder während Virus-Infektionen. Ein bronchialer Provokationstest wird meist durchgeführt, um bei Patienten mit unklaren Atemwegssymptomen zusätzliche Hinweise auf Asthma zu erlangen [1,2]. Es gibt eine Vielzahl von Techniken, mit denen ein solcher Provokationstest durchgeführt werden kann. Zum einen können verschiedene chemische Substanzen als unspezi- 


\begin{tabular}{|c|c|c|c|c|}
\hline Parameter & Mittel & Standabw. & Min & Max \\
\hline Geschlecht & $20 \mathrm{w} / 10 \mathrm{~m}$ & & & \\
\hline Alter (Jahre) & 30 & 11 & 19 & 61 \\
\hline Größe (cm) & 170 & 9 & 158 & 186 \\
\hline Gewicht (kg) & 66 & 11 & 50 & 89 \\
\hline $\mathrm{FEV}_{1}$ & 3,8 & 0,9 & 2,0 & 5,2 \\
\hline $\mathrm{FEV}_{1} \%$ pred & 106 & 10 & 84 & 134 \\
\hline VC (L) & 4,4 & 1,0 & 2,7 & 6,6 \\
\hline VC\%pred & 106 & 11 & 81 & 130 \\
\hline sRaw & 0,66 & 0,24 & 0,29 & 1,28 \\
\hline
\end{tabular}

Tab. 1 Anthropometrische und Lungenfunktions-Daten des Studienkollektiv fischer Reiz eingesetzt werden [3], zum anderen können bei unspezifischer Provokation unterschiedlichste Inhalationstechniken verwendet werden. Eine Methode zur Provokation wurde sowohl von der „American Thoracic Society“ als auch von der „European Respiratory Society“ empfohlen: Die Dosimeter-Methode $[3,4]$. Bei dieser Methode werden in jeder Provokationsstufe 5 Aerosol-Boli verabreicht. Dabei wird die Konzentration der provozierenden Substanz von Stufe zu Stufe erhöht. Allerdings hat diese Methode den Nachteil, dass in der Klinik unterschiedlich konzentrierte Lösungen der provozierenden Substanz bereitgehalten werden müssen. Es wurden daher Modifikationen an der Technik vorgenommen, die es erlauben, nur eine einzige Lösung zu verwenden [2]. Die Provokationstechnik, die in Deutschland weit verbreitet ist, wurde mit der Dosimeter-Technik kombiniert, und ist seit einiger Zeit als kommerzielles Gerät erhältlich - das Viasys-Jäger-APS. Dieses Gerät ist in Deutschland in vielen Krankenhäusern und pneumologischen Praxen im Einsatz. Bei diesem Gerät wird die provozierende Substanz als Bolus während einer freien Inhalation verabreicht. Durch Erhöhung der Zahl der Boli, die pro Provokationsstufe verabreicht wird, wird die Provokationsdosis gesteigert.

Seit einigen Jahren wird ein neues Konzept zur Verabreichung pharmazeutischer Aerosole wissenschaftlich untersucht, das Konzept kontrollierter Inhalation [5]. Dieses Konzept beruht auf der Beobachtung, dass die Dosisvariabilität bei der Verabreichung inhalierter Medikamente zu einem hohen Prozentsatz auf Unterschiede im Atemmanöver beruht, und dass diese Variabilität reduziert werden kann, wenn das Atemmanöver kontrolliert wird. Darüber hinaus wurde gezeigt, dass es möglich ist, das Atemmanöver so auszuwählen, dass eine optimale, das heißt möglichst große Deposition, in der Targetregion des Atemtraktes erzielt werden kann $[5,6,7,8,9,10]$. Mit der kommerziellen Verfügbarkeit eines Gerätes zur Durchführung von kontrollierter Inhalation (AKITA - Activaero GmbH, Gemünden) in der klinischen Praxis ist es möglich geworden, auch für die bronchiale Provokation das Konzept kontrollierter Inhalation in Betracht zu ziehen. Dieses Gerät würde es auch kleineren Praxen ermöglichen, bronchiale Provokationstests auf hohem Standard durchzuführen. Dabei wird die provozierende Substanz ebenfalls als Bolus verabreicht, und die Dosis durch Erhöhung der Boluszahl pro Provokationstufe erhöht. Allerdings erfolgt die Inhalation mit standardisiertem Atemmanöver.

Bevor ein Einsatz des AKITA-Gerätes für bronchiale Provokationstests erwogen werden kann, ist es notwendig, diese Methode mit einer etablierten Methode zu vergleichen und zu validieren. In der vorliegenden Studie wurde die bronchiale Provokation mit Methacholin und der Viasys-Jäger-APS Methode mit der AKITAMethode verglichen. Untersucht wurden 30 Personen mit atopischer Anamnese, jedoch ohne Hinweis auf bestehendes Asthma. In diesem Kollektiv wurde die Prävalenz eines positiven Tester- gebnisses (nach der Literatur 30\% [1,11]) als Zielkriterium mit beiden Methoden bestimmt, und die Ergebnisse wurden miteinander verglichen.

\section{Methoden}

\section{Studienkollektiv}

30 Probanden (20 Frauen, 10 Männer) mit Atopie-Anamnese nahmen an der Studie teil. Die Personen litten entweder unter allergischer Rhinitis, allergischer Konjunktivitis, oder allergischem Ekzem, wiesen aber keine anamnestischen Zeichen eines Asthmas oder sonstiger Lungenerkrankungen auf. Das mittlere Alter lag bei $30 \pm 11$ Jahren, und reichte von 19 bis 61 Jahre. Die anthropometrischen und Lungenfunktionsdaten des Studienkollektivs sind in Tab. 1 zusammengefasst. Die häufigste Begleitmedikation waren Kontrazeptiva (10 Probandinnen) und Antihypertonika (2 Probanden). Ein Patient wurde gegen Cholesterinämie behandelt, eine Patientin war insulinpflichtig. Die Begleitmedikation änderte sich während des Studienzeitraumes nicht. Das Studienprotokoll wurde von der Ethikkommission der Bayerischen Landesärztekammer München auf ethische Unbedenklichkeit geprüft und den zuständigen Behörden gemeldet.

\section{Provokation mit dem APS}

Der Viasys-Jäger-APS ist ein kommerzielles Gerät, das mit einem Bodyplethysmographen oder Spirometer gekoppelt ist. Das Gerät beruht auf einer modifizierten Dosimeter-Technik, bei der Methacholinchlorid in nur einer einzigen Konzentration eingesetzt wird, und die Dosis dadurch variiert wird, dass die Zahl der verabreichten Aerosol-Boli von Stufe zu Stufe erhöht, beziehungsweise die Breite des Aerosolbolus verändert wird. Dazu wurde der Vernebler (Medic Aid, Sidestream, aerodynamischer Massen-Mediandurchmesser der Teilchen MMAD = 3,7 $\mu \mathrm{m}$ ) des APS-Systems mit einer Lösung befüllt, die $16 \mathrm{mg} / \mathrm{ml}$ Methacholinchlorid enthält. Tab. 2 fasst die Parameter der maximal fünf Provokationsstufen zusammen. Es wird eine hoch konzentrierte Methacholinlösung verwendet um zu ermöglichen, dass nur wenige Boli pro Provokationsstufe verabreicht werden müssen. Während der Verabreichung des Methacholin-Bolus wurde der Proband angehalten, langsam und tief zu inhalieren. Nach Inhalation von medikamentenfreier Luft über $0,2 \mathrm{~s}$ wird für $1 \mathrm{~s}$ der Vernebler gestartet und Aerosol verabreicht. Vor Beginn der Provokation und jeweils 2 Minuten nach Abschluss jeder Provokationsstufe wurde eine Bodyplethysmographie und Spirometrie durchgeführt. Lag der gemessene $\mathrm{FEV}_{1}$-Wert dabei unter $80 \%$ des Ausgangswertes, wurde die Provokation abgebrochen, war dies nicht der Fall, so wurde mit der nächsten Stufe fortgefahren. Alle 5 Provokationsstufen führten zu einer maximalen kumulativen Methacholindosis von $777 \mu \mathrm{g}$. 


\begin{tabular}{lllllll} 
Stufe & Substanz & $\begin{array}{l}\text { Konz. } \\
(\mathbf{m g} / \mathbf{m l})\end{array}$ & $\begin{array}{l}\text { Verneblungs- } \\
\text { zeit }(\mathbf{s})\end{array}$ & $\begin{array}{l}\text { Zahl der } \\
\text { Atemzüge }\end{array}$ & $\begin{array}{l}\text { Dosis pro } \\
\text { Atemzug }(\mu \mathbf{g})\end{array}$ & $\begin{array}{l}\text { Kum. Dosis } \\
(\mu \mathbf{g})\end{array}$ \\
\hline 0 & $\mathrm{NaCl}$ & 9 & 0,60 & 5 & & \\
\hline 1 & Methach. & 16 & 0,29 & 2 & 25 & 75 \\
\hline 2 & Methach. & 16 & 0,59 & 2 & 50 & 175 \\
\hline 3 & Methach. & 16 & 0,59 & 4 & 100 & 376 \\
\hline 4 & Methach. & 16 & 0,59 & 8 & 201 & 777 \\
\hline 5 & Methach. & 16 & 0,94 & 10 & 401 & \\
\hline
\end{tabular}

Tab. 2 Zusammenfassung der 5 Provokationstufen beim APSSystem

\begin{tabular}{|c|c|c|c|c|c|c|}
\hline Stufe & Substanz & $\begin{array}{l}\text { Konz. } \\
\text { (mg/ml) }\end{array}$ & $\begin{array}{l}\text { Verneblungs- } \\
\text { zeit (s) }\end{array}$ & $\begin{array}{l}\text { Anzahl von } \\
\text { Atemzügen }\end{array}$ & $\begin{array}{l}\text { Dosis pro } \\
\text { Atemzug }(\mu \mathrm{g})\end{array}$ & $\begin{array}{l}\text { kum. Dosis } \\
(\mu \mathrm{g})\end{array}$ \\
\hline & $\mathrm{NaCl}$ & 9 & 1,0 & 1 & 69 & \\
\hline 1 & Methach. & 3,3 & 1,0 & 1 & 25 & 25 \\
\hline 2 & Methach. & 3,3 & 1,0 & 2 & 50 & 75 \\
\hline 3 & Methach. & 3,3 & 1,0 & 4 & 100 & 176 \\
\hline 4 & Methach. & 3,3 & 1,0 & 8 & 201 & 377 \\
\hline 5 & Methach. & 3,3 & 1,0 & 16 & 402 & 778 \\
\hline
\end{tabular}
Tab. 3 Zusammenfassung der 5 Provokationstufen beim AKI- TA-System

\section{Provokation mit der AKITA}

Das AKITA-System ist ein neues, zur Inhalation von Medikamenten zugelassenes Inhalationsgerät, bei dem das Konzept kontrollierter Inhalationen verwirklicht ist. Dieses Gerät besteht aus einem kommerziellen Vernebler (Pari-LC Star, MMAD = 3,8 $\mu \mathrm{m}$ ) und einem computergesteuerten Kompressor, der getrennt sowohl die Pressluft für die Verneblerdüse, als auch die Zusatzluft liefert. Damit kann ein Aerosolbolus vorgewählter Breite zu einem vorbestimmten Zeitpunkt eines Atemzugs definierter Tiefe appliziert werden. Der Atemfluss, mit dem diese Medikamentenapplikation erfolgt, kann ebenso wie die Zahl der Atemzüge und eine Atempause vorgewählt werden. Das AKITA-System wird mit dem gewählten Atemmanöver über eine Smart-Card programmiert. Zur Durchführung einer bronchialen Provokation wurde der Vernebler des AKITA-Systems mit einer Lösung befüllt, die $3 \mathrm{mg} / \mathrm{ml}$ Methacholin enthielt (Provokit-Lindopharm). Die Inhalation des Medikaments erfolgte in einem Atemzug mit einem Volumen von 0,8 L (Atemfluss: $200 \mathrm{~cm}^{3} / \mathrm{s}$ ). Die Breite des Aerosolbolus lag bei $200 \mathrm{~cm}^{3}$, so dass nach Bolusapplikation $600 \mathrm{~cm}^{3}$ wirkstofffreie Luft inhaliert wurden. Die genauen Parameter jeder Provokationsstufe sind in Tab. 3 zusammengefasst. Die maximale kumulative Methacholindosis lag bei dieser Methode bei $778 \mu \mathrm{g}$. Die Messung der Lungenfunktion, sowie der Abbruch der Provokation erfolgten analog zur APS-Methode.

\section{Lungenfunktion}

Die Messung der bodyplethysmographischen und spirometrischen Lungenfunktion erfolgte mit einem Viasys-Jäger-Masterlab. Die folgenden Parameter wurden bestimmt: Vitalkapazität (VC), forciertes Volumen in einer Sekunde $\left(\mathrm{FEV}_{1}\right)$, intrathorakales Gasvolumen (ITGV), totale Lungenkapazität (TLC), sowie der spezifische Atemwegswiderstand $\left(\mathrm{sR}_{\mathrm{AW}}\right)$. Als Vergleichswerte wurden die EGKS-Referenzwerte herangezogen [12]. Bei Einschleusung jedes Probanden und vor jeder Provokation wurden alle oben genannten Parameter bestimmt. Zwei Minuten nach jeder Provokationsstufe wurden $\mathrm{FEV}_{1}$ und $\mathrm{sR}_{\mathrm{AW}}$ gemessen, und die Änderung dieser beiden Parameter berechnet. Ein Provokationstest wurde als positiv gewertet, wenn im Verlauf der 5 Provokationsstufen der $\mathrm{FEV}_{1}$ unter $80 \%$ des Ausgangswertes absank. In einer zweiten Auswertung wurde eine 100\%ige Zunahme des $s R_{A W}$ als positives Testergebnis gewertet.

\section{Studienablauf}

Alle Probanden wurden mit beiden Provokationstechniken untersucht, und jeweils als $\mathrm{FEV}_{1}$-positiv bzw. -negativ sowie als $\mathrm{sR}_{\mathrm{AW}}$-positiv bzw. -negativ klassifiziert. Die Reihenfolge, in der die beiden Methoden durchgeführt wurden, war randomisiert. Zwischen beiden Untersuchungen lag ein Zeitraum von 3 bis maximal 10 Tagen. Reagierte ein Proband mit einem Abfall des $\mathrm{FEV}_{1}$, so erfolgte, falls erforderlich, eine Broncholyse mit $200 \mu \mathrm{g}$ Salbutamol. Zur Beurteilung der Sicherheit beider Methoden wurde vor und nach der Provokation eine Blutdruckmessung durchgeführt, und unerwünschte Ereignisse und Nebenwirkungen registriert.

\section{Statistik}

Unterschiede zwischen den Mittelwerten von Messgrößen wurden mithilfe des Student-T-Test (SAS für Windows, Version 9.1.3), Unterschiede in den Prävalenzen von positiven Ergebnissen mit dem McNemar-Test auf statistische Signifikanz geprüft.

\section{Ergebnisse}

Betrachtet man die Zahl der Personen, die mit einem mehr als $20 \%$ igem Abfall des $\mathrm{FEV}_{1}$ auf bronchiale Provokation reagierten, so wurden bei der APS-Methode 8 und bei der AKITA 9 positive Befunde beobachtet (Tab. 4). Dieser geringe Unterschied ist statistisch nicht signifikant $(\mathrm{p}=1)$.

Verwendet man einen $100 \%$ igen Anstieg des $\mathrm{sR}_{\mathrm{AW}}$ als Indikator für ein positives Testergebnis, so findet man deutlich höhere Werte: 17 Probanden bei Inhalation mit der AKITA und 18 Probanden bei Inhalation mit dem APS mussten als positiv eingestuft werden (Tab. 5). Dieser geringe Unterschied zwischen beiden Methoden war auch hier statistisch nicht signifikant $(p=1)$. Allerdings hat sich gezeigt, dass zwischen den Ergebnissen der beiden Methoden Diskrepanzen bestehen: Bei 3 Probanden, die im Hinblick auf einen 20\%-Abfall des $\mathrm{FEV}_{1}$ mit der AKITA positiv reagierten, wurde mit dem APS ein negatives Ergebnis gefunden; bei 2 Probanden, die mit dem APS ein positives Ergebnis zeigten, war der Test mit der AKITA negativ. Bei 25 von 30 Fällen stimmte das Ergebnis (positiv oder negativ) zwischen beiden Methoden überein. Betrachtet man den $\mathrm{sR}_{\mathrm{AW}}$, so findet man, dass bei 4 Per- 
Tab. 4 Anzahl positiver und negativer Testergebnisse für beide Provokationstechniken und den Parameter FEV 1

\begin{tabular}{lccl} 
FEV $_{\mathbf{1}}$ & AKITA & APS & Summe \\
positiv & 9 & 8 & 17 \\
\hline negativ & 21 & 22 & 43 \\
\hline Summe & 30 & 30 &
\end{tabular}

Tab. 5 Anzahl positiver und negativer Testergebnisse für beide Provokationstechniken und den Parameter sR $\mathrm{AW}_{\mathrm{AW}}$

\begin{tabular}{|llll|}
\hline $\mathbf{S R}_{\text {AW }}$ & AKITA & APS & Summe \\
\hline positiv & 17 & 18 & 35 \\
\hline negativ & 13 & 12 & 25 \\
\hline Summe & 30 & 30 & \\
\hline
\end{tabular}

Tab. 6 Individuelle Auflistung der Ergebnisse der Provokationstests bei den einzelnen Probanden. Ein grau markiertes mit „, “ bezeichnetes Feld kennzeichnet ein positives Ergebnis

\begin{tabular}{|c|c|c|c|c|}
\hline Proband & $\begin{array}{l}\text { AKITA } \\
\text { FEV }_{1} \\
\text { Ergebnis }^{2}\end{array}$ & $\begin{array}{l}\mathbf{S R}_{\mathrm{AW}} \\
\text { Ergebnis }\end{array}$ & $\begin{array}{l}\text { APS } \\
\text { FEV }_{1} \\
\text { Ergebnis }^{2}\end{array}$ & $\begin{array}{l}\mathbf{s R}_{\text {AW }} \\
\text { Ergebnis }\end{array}$ \\
\hline 1 & - & + & - & - \\
\hline 2 & + & + & - & - \\
\hline 3 & - & - & - & - \\
\hline 4 & - & + & + & + \\
\hline 5 & - & - & + & - \\
\hline 6 & - & + & - & + \\
\hline 7 & + & + & + & + \\
\hline 8 & - & - & - & - \\
\hline 9 & + & + & - & - \\
\hline 10 & - & + & - & - \\
\hline 11 & - & - & - & + \\
\hline 12 & - & - & - & + \\
\hline 13 & - & + & - & + \\
\hline 14 & - & - & - & - \\
\hline 15 & + & + & + & + \\
\hline 16 & - & + & - & + \\
\hline 17 & + & + & + & + \\
\hline 18 & - & - & - & - \\
\hline 19 & - & - & - & + \\
\hline 20 & - & + & - & + \\
\hline 21 & - & + & - & + \\
\hline 22 & - & + & - & + \\
\hline 23 & + & - & - & + \\
\hline 24 & - & - & - & - \\
\hline 25 & - & - & - & - \\
\hline 26 & + & + & + & + \\
\hline 27 & - & - & - & + \\
\hline 28 & - & - & - & - \\
\hline 29 & + & + & + & + \\
\hline 30 & + & + & + & + \\
\hline
\end{tabular}

Tab. 7 Mittlere kumulative Provokationsdosis, die zu einem 20\% Abfall des $\mathrm{FEV}_{1}$ (PD20) bzw. eines $100 \%$ Anstieg des sR $\mathrm{AW}_{\text {AW }}$ (PD100) bei Personen mit positivem Testergebnis mit beiden Methoden führten. $p$ bezeichnet die Irrtumswahrscheinlichkeit für die Signifikanz des Gruppenunterschiedes

\begin{tabular}{|l|cccc|}
\hline & $\begin{array}{l}\text { PD20 }(\mu \mathbf{g}) \\
\text { Mittel }\end{array}$ & St.abw. & $\begin{array}{l}\text { PD100 }(\mu \mathbf{g}) \\
\text { Mittel }\end{array}$ & St.abw. \\
\hline AKITA & 488,67 & 198,30 & 350,79 & 191,82 \\
\hline APS & 415,10 & 107,43 & 325,11 & 222,98 \\
\hline P & 0,35 & & 0,72 & \\
\hline
\end{tabular}

sonen mit positivem AKITA-Befund, ein negatives APS-Ergebnis vorlag, und bei 5 Personen mit positivem APS-Befund war das AKITA-Ergebnis negativ. Bei 21 der 30 Fälle stimmte das Ergebnis überein (Tab. 6).

Bei den Personen, die positiv auf den Provokationstest reagierten, lag die kumulative Methacholin-Dosis für den 20\%-igen Abfall des $\mathrm{FEV}_{1}$ im Mittel bei $489 \mu \mathrm{g}$ (AKITA) bzw. $415 \mu \mathrm{g}$ (APS) (Tab. 7). Auch bei diesem Parameter konnte für die beiden Provokationsmethoden kein statistisch signifikanter Unterschied gefunden werden.

Die Sicherheit der neuen Provokationstechnik mit der AKITA wurde anhand der Häufigkeit von unerwünschten Ereignissen und von Blutdruckänderungen im Vergleich zur APS-Methode abgeschätzt.

Nennenswerte Nebenwirkungen, abgesehen von Hustenreiz und leichten Kopfschmerzen, wurden bei dieser Studie bei beiden Methoden nicht beobachtet. Änderungen des Blutdrucks und der Pulsfrequenz durch den Provokationstest waren bei beiden Methoden im Mittel vernachlässigbar gering, und statistisch nicht von Null unterscheidbar (Tab. 8).

\section{Diskussion}

In dieser Studie konnte gezeigt werden, dass die beiden untersuchten Methoden zur Durchführung bronchialer Provokationstests mit Methacholin, die etablierte APS-Methode und die neu entwickelte AKITA-Methode, gleichwertig sind. Zwischen beiden Methoden konnten keine Unterschiede gefunden werden: Weder bei der Häufigkeit positiver Befunde, bei der mittleren Provokationsdosis der positiv reagierenden Probanden, noch bei sicherheitsrelevanten Parametern wie Blutdruck und Pulsfrequenz. Auch die Annahme einer 30\%-Rate von positiven Testergebnissen in dem gewählten Personenkollektiv, die bei Studienplanung und Fallzahlabschätzung gemacht wurde, stellte sich als richtig heraus. Allerdings zeigte sich, dass trotz übereinstimmender Prävalenz positiver Befunde, die individuellen Ergebnisse der einzelnen Personen nicht in allen Fällen übereinstimmten. Im Falle des $\mathrm{FEV}_{1}$ als Indikator für eine positive Reaktion zeigte sich, dass 25 von 30 Ergebnissen übereinstimmten. Im Falle des $s R_{\text {AW }}$ waren es sogar nur 21 von 30 Fällen. Dieser Befund ist nicht einfach zu verstehen:

- Da zwischen beiden bronchialen Provokationen ein Zeitabstand von bis zu 10 Tagen lag, ist es vorstellbar, dass Änderungen in der individuellen Suszeptibilität gegenüber Methacholin Ursache für eine derartige Diskrepanz waren.

- Auch Unterschiede im Atemmanöver zwischen verschiedenen Personen, die mit dem APS-Gerät möglich sind, können derartige Befunde prinzipiell verursachen. Solche Unterschiede im Atemmanöver können dazu führen, dass einzelne Personen höheren oder geringeren Methacholin-Dosen ausgesetzt waren, als andere Personen. Damit kann es zu „falsch positiven“ oder „falsch negativen“ Ergebnissen kommen.

Dieses Ergebnis verdeutlicht eines der Hauptprobleme der bronchialen Provokation: Das Fehlen eines zuverlässigen „Gold Standards“. Es gibt keine Technik, mit der „wahr positive“ und „wahr negative“ Testergebnisse eindeutig definiert werden können. Die Schwelle für positive Ergebnisse ist letztlich willkürlich. Dies zeigt sich insbesondere darin, dass man, zieht man $s R_{A W}$ als Indikator für ein positives Testergebnis heran, etwa doppelt so viele positive Ergebnisse findet. Da darüber hinaus der diagnostische und prospektive Wert bronchialer Provokationstest kontrovers 


\begin{tabular}{|c|c|c|c|c|c|c|c|c|}
\hline & & $\begin{array}{l}\text { vor Provo } \\
\text { Systole } \\
(\mathrm{mmHg})\end{array}$ & $\begin{array}{l}\text { Diastole } \\
(\mathrm{mmHg})\end{array}$ & $\begin{array}{l}\text { Puls } \\
\text { (1/min) }\end{array}$ & $\begin{array}{l}\text { nach Prov } \\
\text { Systole } \\
(\mathrm{mmHg})\end{array}$ & $\begin{array}{l}\text { Diastole } \\
\text { (mmHg) }\end{array}$ & $\begin{array}{l}\text { Puls } \\
(1 / \mathrm{min})\end{array}$ & $\begin{array}{l}\text { Tab. } 8 \text { Blutdruck und Pulsfre- } \\
\text { quenz vor und nach bronchialer } \\
\text { Provokation mit beiden Me- } \\
\text { thoden }\end{array}$ \\
\hline \multirow[t]{2}{*}{ AKITA } & Mittelwert & 123 & 78 & 75 & 121 & 76 & 77 & \\
\hline & Std.Abw. & 17 & 9 & 9 & 17 & 10 & 10 & \\
\hline \multirow[t]{2}{*}{ APS } & Mittelwert & 118 & 75 & 73 & 119 & 77 & 76 & \\
\hline & Std.Abw & 12 & 8 & 12 & 14 & 8 & 12 & \\
\hline
\end{tabular}

diskutiert wird [1], können auch schwerlich unangreifbare prospektive Studien entworfen werden, die die Qualität eines Provokationsverfahren an ihrem prospektiven Wert misst: Weder der Wert des bronchialen Provokationstests als Prädiktor für zukünftiges Asthma, noch sein Wert zur Definition eines Krankheitsschweregrades oder des Inflammationsstatus sind unumstritten, und sind daher als Endpunkte solcher Studien möglicherweise nicht geeignet.

Aufgrund dieser Problematik ist es sicherlich nicht sinnvoll, die in dieser Studie gefundenen Diskrepanzen zwischen beiden Provokationsmethoden zu überbewerten. Allein die Tatsache, dass beide Methoden zur selben Prävalenz von positiven Befunden führen, kann als Hinweis gewertet werden, dass auch die Provokation mit der AKITA für eine Anwendung in der klinischen Praxis geeignet ist.

Ein weiteres interessantes Ergebnis dieser Studie spiegelt sich in der Tatsache, dass etwa doppelt so viele Probanden mit einem $100 \%$ igen Anstieg des $\mathrm{rR}_{\mathrm{AW}}$ reagierten, als mit einem 20\%-igen

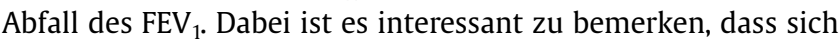
bei einigen Personen, die eine deutliche Zunahme des $\mathrm{sR}_{\mathrm{AW}}$ zeigten, keinerlei Veränderung des $\mathrm{FEV}_{1}$ zu beobachten war. Dieser Befund macht deutlich, dass beide Messgrößen deutlich verschiedene Aspekte der Lungenmechanik widerspiegeln, und eher komplementäre als redundante Informationen liefern. Während der $s R_{A W}$ bei Ruheatmung gemessen wird, und vom statischen Kaliber auch kleiner Atemwege beeinflusst wird, spiegelt der $\mathrm{FEV}_{1}$, gemessen bei forcierter Exspiration, auch beim gesunden Probanden zum Teil die elastischen Eigenschaften kleiner Atemwege (exspiratorischer Kollaps), weniger jedoch deren Kaliber, wider. Damit könnte spekuliert werden, dass der Ort der induzierten Obstruktion bei bronchialer Provokation bei $\mathrm{FEV}_{1}$-Respondern und $\mathrm{sR}_{\mathrm{AW}}$-Respondern unterschiedlich von einander ist.

\section{Schlussfolgerungen}

In dieser Studie konnte gezeigt werden, dass die beiden untersuchten Methoden zur Durchführung bronchialer Provokationstests mit Methacholin, die APS-Methode und AKITA-Methode, im Hinblick auf Prävalenz positiver Ergebnisse, mittlerer Provokationsdosis bei Respondern, Blutdruck- und Pulsfrequenz-Änderung, sowie dem Auftreten von unerwünschten Ereignissen und Nebenwirkungen gleichwertig sind. Damit kann die Methode der bronchialen Provokation mit kontrollierter Inhalation als für die klinische Praxis geeignet eingeschätzt werden.

\section{Literatur}

1 Smith L, McFadden ER Jr: Bronchial hyperreactivity revisited. Ann Allergy Asthma Immunol 1995; 74 (6): 454- 469

2 Klein G: Empfehlungen zur Durchführung bronchialer Provokationstests mit pharmakologischen Substanzen. Medizinische Klinik 1997; 92: $458-463$

3 Sterk PJ, Fabbri LM, Quanjer PHet al: Airway Responsiveness: Standardized challenge testing with pharmacological, physical and sensitizing stimuli in adults. Eur Respir J 1993; 6: 53-83

4 Crapo RO, Casaburi R, Coates ALet al: Guidelines for methacholine and exercise challenge testing-1999. This official statement of the American Thoracic Society was adopted by the ATS Board of Directors, July 1999. Am J Respir Crit Care Med 2000; 161 (1): 309-329

5 Brand P, Friemel I, Meyer Tet al: Total deposition of therapeutic particles during spontaneous and controlled inhalations. J Pharmaceut Sci 2000; 89: 724-731

6 Brand P, Enriquez MM, Meyer Tet al: Neue Strategien zur kontrollierten Inhalation von $\alpha 1$-Antitrypsin. Pneumologie 2003; 57: 153- 158

7 Scheuch G, Meyer T, Müllinger Bet al: Controlled inhalation improves peripheral lung deposition. J Aerosol Med 2001; 14: 388 - 388

8 Brand P, Meyer T, Häussermann Set al: Optimum peripheral drug deposition in patients with cystic fibrosis. J Aerosol Med 2005; 18 (1): $45-54$

9 Brand P, Beckmann H, Enriquez MMet al: Peripheral Deposition of $\alpha 1$-Protease Inhibitor Using Commercial Inhalation Devices. Eur Resp J 2003; 22: 263 - 267

10 Griese M, Ramakers J, Krasselt Aet al: Improvement of alveolar glutathione and lung function but not oxidative state in cystic fibrosis. Am J Respir Crit Care Med 2004; 169 (7): 822 - 828

11 Prieto L, Berto JM, Gutierrez V: Airway responsiveness to methacholine and risk of asthma in patients with allergic rhinitis. Ann Allergy 1994; 72 (6): $534-539$

12 Quanjer PH, Tammeling GJ, Cotes JEet al: Lung Volumes and Forced Ventilatory Flows. Eur Respir J 1993; 6: 5-40 